\title{
OBJETIVOS DE SELECCIÓN PARA LA CRIANZA DE ALPACAS HUACAYA BAJO DOS ESCENARIOS ECONÓMICOS EN LA SIERRA CENTRAL DEL PERÚ
}

\section{SELECTION OBJECTIVES FOR HUACAYA ALPACA BREEDING UNDER TWO ECONOMIC SCENARIOS IN THE CENTRAL HIGHLANDS OF PERU}

\author{
Julissa Raquel Candio Lopez ${ }^{1,2}$ y Gustavo Augusto Gutiérrez Reynoso ${ }^{1,3}$
}

\begin{abstract}
Resumen
El presente trabajo de investigación consistió en determinar los objetivos de selección para la crianza de alpacas bajo dos escenarios económicos en la Cooperativa Comunal San Pedro de Racco. El estudio se realizó en tres etapas: En la primera se describió la crianza de alpacas, en la segunda se elaboró la ecuación de ganancias, y en la tercera se realizó el cálculo de los valores relativos económicos por derivación parcial y se definieron los objetivos de selección. Los escenarios económicos 1 y 2 fueron definidos por la forma de pago de la fibra. Los valores relativos económicos del peso de vellón (PVL) y peso de vellón a la primera esquila (PVL1) fueron S/10.6 y S/5.85, respectivamente, valores que fueron iguales en ambos escenarios económicos. En el escenario económico 2, el diámetro de fibra (DF) obtuvo un valor de -S/2.12. El objetivo de selección por escenarios económicos fue definido por PVL, PVL1 y peso vivo a la primera esquila (P1E) para el escenario 1; y por PVL, PVL1 y DF para el escenario 2. Se concluyó que el pago por fibra categorizada favoreció el valor relativo económico del diámetro de fibra y diámetro de fibra a la primera esquila, contribuyendo al afinamiento de los vellones; mientras que el peso de vellón fue el carácter de mayor valor relativo económico en los objetivos de selección de los escenarios económicos 1 y 2.
\end{abstract}

Palabras clave: alpaca, objetivo de selección, valor relativo económico.

\begin{abstract}
The research consisted in determining the selection objectives for raising alpacas under two economic scenarios at the San Pedro de Racco Community Cooperative. The study was carried out in three stages: The first one described the alpaca's breeding system, in the second one the elaboration of the profit equation was done, and in the third one the relative economic values by partial derivation were calculated and the selection objectives were determined. Economic scenarios 1 and 2 were defined by the payment method of the fiber. The relative economic values of fleece weight (FW) and fleece weight at the first shearing (FW1) were S/10.6 and S/5.85, respectively, values which were equal for both economic scenarios. The fiber diameter (FD) obtained values of $-\mathrm{S} / 2.12$ in economic scenario 2 . The selection objective by economic scenarios was defined by FW, FW1, and live weight at first shearing (LW1S) for scenario 1; and by FW, FW1, and FD for scenario 2. It was concluded that the payment by categorized fiber favored the relative economic value of the fiber diameter and fiber diameter at the first shearing, contributing to the thinning of the fleeces; while the fleece weight was the character of greatest relative economic value in the selection objectives of economic scenarios 1 and 2.
\end{abstract}

Key words: alpaca, selection objective, relative economic value.

\section{Introducción}

Perú tenía una población de 3685516 de alpacas (Vicugna pacos (Linnaeus, 1758)) hasta antes del 2012 (INEI, 2012) y su crianza es, y ha sido, una actividad de gran importancia económica para el poblador de la zona altoandina. Esta crianza se desarrolla por encima de los $3500 \mathrm{msnm}$ donde la agricultura y la crianza de otras especies, como los vacunos y ovinos, no alcanzan altos niveles productivos debido a las condiciones climáticas adversas de la puna. La cría de alpacas ofrece: a) producción de fibra para los mercados nacional y mundial, b) producción de carne para el mercado nacional, c) reproductores y pieles como productos secundarios o subproductos (Germaná et al., 2016; Gutiérrez et al., 2019).
La producción de fibra en el Perú tuvo un incremento de 1.2 mil toneladas entre los años 2004 y 2013 (MINAGRI, 2015), sin embargo, aproximadamente el $50 \%$ de la producción de fibra sigue siendo gruesa, con una finura superior a las 26 micras $(\mu)$, generando bajos ingresos a los productores alpaqueros. La producción de carne de alpaca en el año 2013 fue de 12.2 mil toneladas, mostrando un incremento de 2.8 mil toneladas entre los años 2004 al 2013 (MINAGRI, 2015). La carne de alpaca es un producto que deriva de la saca o descarte de alpacas con características poco deseables para la producción de fibra, siendo destinada al autoconsumo y venta local (Salvá et al., 2009). 
En este contexto, según estadísticas oficiales, la producción de fibra y carne ha mostrado un incremento considerable; sin embargo, esto no está relacionado a una mejora en la calidad y precios de la fibra y carne de alpaca. Es así que en los últimos años hubo esfuerzos por parte del estado a través del MINAGRI e INIA, gobiernos regionales $y$ organismos no gubernamentales, en apoyar programas de mejoramiento genético de alpacas; sin embargo, no consideraron los posibles escenarios económicos en los que se ven involucrados los productores de alpacas debido a la forma de pago y calidad de la fibra (Gutiérrez et al., 2019).

Objetivo de selección es una herramienta que permite estimar los caracteres fenotípicos que serán utilizados en programas de mejoramiento animal y permitirá seleccionar animales por su calidad y valor económico (Nielsen et al., 2014). En el caso de la crianza de alpacas, la definición de un objetivo de selección que incluya el análisis de los posibles escenarios económicos, según la forma de pago de la fibra, podría constituir una herramienta para ser aplicada en programas de mejora genética, con lo cual el productor alpaquero tendrá mayores posibilidades de incrementar sus ingresos.

El propósito del presente estudio fue determinar los objetivos de selección para la crianza de alpacas de la Cooperativa Comunal San Pedro de Racco (Pasco/Perú) bajo dos posibles escenarios económicos considerando en ambos casos la venta de animales en pie, pero diferenciando la forma de venta de la fibra al barrer o categorizada. Los objetivos específicos del estudio fueron describir la crianza de alpacas mediante los índices técnicos, ingresos y costos de las principales actividades de manejo de alpacas, y finalmente, con la información generada, establecer la ecuación de ganancias y determinar los valores relativos económicos para la definición de los objetivos de selección para los dos escenarios económicos.

\section{Materiales y métodos}

Área de estudio

La presente investigación se realizó en la Cooperativa Comunal San Pedro de Racco (CCSPR), localizada en el Distrito Simón Bolívar, en la Región Pasco (Perú), a una elevación entre los 4253 a 4446 msnm (Figura 1). La zona se caracteriza por presentar una temperatura media anual máxima de 7.2 ${ }^{\circ} \mathrm{C}$ y una media anual mínima de $3{ }^{\circ} \mathrm{C}$. El rango máximo de precipitación oscila entre 1000 a $1819 \mathrm{~mm}$ y el mínimo entre 500.0 a $828.7 \mathrm{~mm}$ por año (SENAMHI, 2015).

La CCSPR, fue constituida el 16 de junio de 1968 al interior de la comunidad campesina San Pedro de Racco, luego de la reforma agraria y reconocida jurídicamente el 25 de junio de 1969, siendo inscrita en la Superintendencia Nacional de Administración Tributaria (SUNAT) con RUC 20131453427. Está conformada por 140 socios de la comunidad campesina y desarrolla la crianza comercial de ovinos, alpacas, llamas y vacunos. La CCSPR maneja 4275.8 hectáreas de pastos naturales en calidad de usufructo; destinando el principal uso de los pastizales a la crianza de ovinos de la raza Corriedale y alpacas Huacaya color blanco.

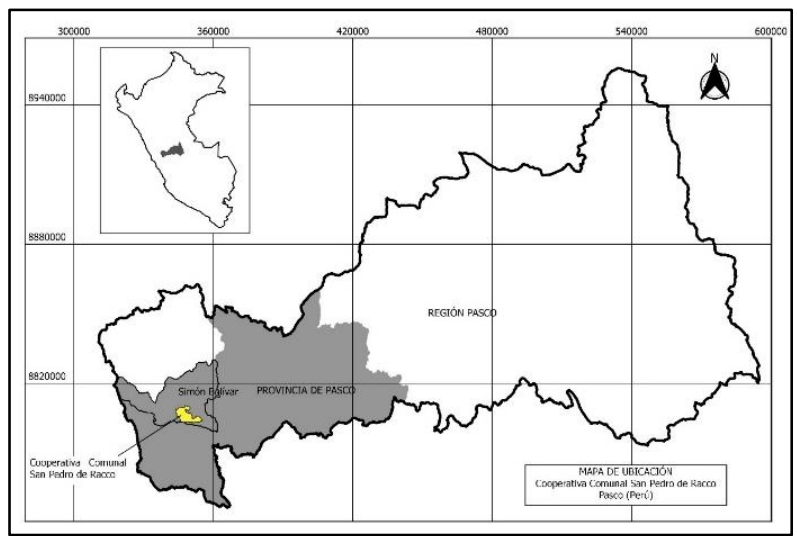

Fuente: Elaboración Propia.

Figura 1. Ubicación de la Cooperativa Comunal San Pedro de Racco.

\section{Animales}

La población de alpacas de la CCSPR correspondiente al periodo 2006-2016 fue en promedio 2565 alpacas y fueron evaluadas según la edad, estado productivo y categorías. Para fines de los cálculos y estimaciones, sólo fue considerada la población de alpacas Huacaya de color blanco, la cual fue dividida en tres categorías: madre, padre y tui. Las categorías padre y madre incluyeron a alpacas hembras y machos mayores de dos años en actividad reproductiva, y la categoría tui correspondió tanto a los tuis menores (alpacas destetadas desde los siete hasta los 14 meses de edad) como los tuis mayores (alpacas desde los 14 hasta los 24 meses de edad).

Colección de datos

La información fue recopilada a partir de los balances ganaderos mensuales, boletas, facturas y balances contables de la CCSPR. Los balances ganaderos mensuales contenían registros de ingresos, movimientos por desbarate, egresos y existencias de fin de mes de alpacas, correspondientes al periodo 20062016. Los balances contables contenían información de los ingresos y costos de producción de alpacas correspondientes al periodo 2006-2016. Las boletas y facturas emitidas para los rubros de venta de fibra de alpaca color blanco, reproductores y animales en pie, fueron 338 para el periodo 2006-2010 y 58 boletas para el periodo 2015-2016.

Etapas de la investigación

a. Descripción de la crianza de alpacas en la CCSPR.

La estimación de los índices técnicos, ingresos y costos, se realizó siguiendo la metodología empleada por Ponzoni (1986) y Bohan et al. (2019). Los índices 
técnicos fueron descritos a partir del capital promedio anual (CPA), mortalidad (en \%), natalidad bruta (NB en \%), natalidad real (NR en \%), saca o descarte (S en $\%$ ), incremento bruto, incremento real y eficiencia ganadera. Los costos de crianza fueron estimados aplicando la técnica de presupuesto parcial con relación al volumen o tamaño de la actividad, determinando así los costos fijos y variables (Workman, 1981).

Los costos fijos fueron expresados en base a una unidad alpaca (UAL) y provienen de las actividades operativas de dirección, administración y servicios comunales. Los costos variables fueron estimados en base a los costos incurridos en la generación de ingresos, incluyendo las actividades de manejo (empadre, parición, destete, esquila y selección de reemplazos). Los costos variables también fueron expresados en base a una UAL para el periodo de un año. El costo de alimentación fue establecido por el pago por calidad de uso del pastizal y mano de obra requerida para el pastoreo del rebaño.

b. Definición de escenarios económicos.

Los escenarios económicos fueron definidos según la forma de pago de la fibra, considerando el pago por fibra al barrer (FB) y el pago por fibra categorizada (FC).

b.1. Escenario económico 1.

Se asumió como fuente principal de ingreso la fibra blanca al barrer y como ingreso secundario la venta de alpacas en pie. El pago por las alpacas en pie se definió en S/250.00 por unidad (S/ símbolo de sol, moneda de Perú), calculado en base a los precios registrados en las boletas y facturas emitidas por la CCSPR. El pago de la fibra blanca se definió en S/10.00 por libra (lb) de FB.

b.2. Escenario económico 2.

Se asumió como fuente de ingreso principal el pago por fibra blanca categorizada y como ingreso secundario la venta de las alpacas en pie. El pago de las alpacas en pie fue establecido por unidad con un precio de S/250.00 por alpaca, sin diferenciación por edad y peso vivo. El pago de la FC fue establecido como se indica en la Fórmula 1.

$$
\mathrm{pFC}=\mathrm{pFB}+(\operatorname{vrCV}-\mathrm{DF}) \times \mathrm{CC}
$$

Fórmula 1. Determinación para el pago por fibra categorizada.

Donde:

pFC : pago por fibra categorizada (en soles/lb); pFB : pago por fibra al barrer $=10.00$ soles $/ \mathrm{lb}$;

vrCV: valor referencial por categoría del vellón;

DF :finura del vellón;

CC : compensación para incentivo económico por micra de finura para los vellones de cada categoría (soles/ $\mu)$;

$(\operatorname{vrCV}-\mathrm{DF}) \times \mathrm{CC}=\mathrm{pCV}$ : pago por categoría del vellón.
El precio base de la FB fue de $\mathrm{S} / 10.00 \cdot \mathrm{lb}^{-1}$. El pago por categoría del vellón (pCV) fue definido según los valores referenciales (vrCV) de Alfonso et al. (2011), quienes establecieron finuras promedio de 20.8, 22.90 y $24.89 \mu$ para las categorías extrafina, fina y semifina, respectivamente. La diferencia entre el vrCV y la finura del vellón (DF) determinó la cantidad de micras para el cálculo del pCV. Asimismo, la compensación (CC) correspondió al incentivo económico por micra de finura para los vellones de cada categoría en el orden de $S / 2.00 \cdot \mu^{-1}$ para la categoría extrafina, $S / 1.00 \cdot \mu^{-1}$ para la categoría fina y $S / 0.50 \cdot \mu^{-1}$ para la categoría semifina. c. Ecuaciones de ganancias.

Las ecuaciones de ganancia fueron formuladas para los escenarios económicos 1 y 2 , en base a los caracteres biológicos con influencia en los ingresos y costos. Los caracteres biológicos fueron diferenciados según la edad y estado fisiológico; además, fueron seleccionados teniendo como referencia los parámetros genéticos de alpacas reportados por Bustinza et al. (1988), Gutiérrez et al. (2008), Cervantes et al. (2010), Gutiérrez (2011), Mamani (2013), Cruz et al. (2015) y Roque (2019). Las ecuaciones de ganancias fueron expresadas mediante el enfoque de maximización del beneficio por animal, según Ponzoni (1986) y Bohan et al. (2019), donde la ganancia se estimó por diferencia entre ingresos y costos.

Asimismo, las ecuaciones de ganancias fueron formuladas utilizando parámetros productivos y reproductivos calculados en base a los índices técnicos del periodo 2007-2010 y 2014-2016 de la CCSPR. Los valores productivos fueron 6 y $3 \mathrm{lb}$ para el peso de vellón en alpacas adultas y tuis, respectivamente. Para el peso vivo se consideró 55, 60 y $35 \mathrm{~kg}$ para madres, padres y tuis de un año de edad. En el caso de la finura se consideró un promedio de 20, 22 y 24 micras para los vellones de las categorías extrafina, fina y semifina, respectivamente. Los valores reproductivos utilizados fueron $65 \%$ de NB, $10 \%$ de mortalidad en crías, $2 \%$ de mortalidad en alpacas adultas y $25 \%$ en tasa de reemplazo.

La ecuación de ganancias del escenario económico 1 es definida según la Fórmula 2.

Los caracteres biológicos relacionados a los tuis fueron la sobrevivencia a la primera esquila (SV), el peso del vellón a la primera esquila (PVL1) y el peso vivo a la primera esquila (P1E). Para la categoría madre y padre, los caracteres fueron el peso de vellón (PVL) $\mathrm{y}$ el peso vivo (PV). 


$$
\begin{aligned}
\mathrm{EC} 1= & \mathrm{NM} \times \mathrm{TN} \times \mathrm{SV}(\mathrm{PVL} 1 \times \mathrm{pf} \\
& +\mathrm{P} 1 \mathrm{E} \times \mathrm{pcb} \times 0.50-\mathrm{ct}) \\
& +(\mathrm{NM}+\mathrm{NP})(\mathrm{PVL} \times \mathrm{pf}) \\
& +\mathrm{NM} \times \mathrm{PV} \times \mathrm{pcb} \times 0.25 \\
& +\mathrm{NP} \times \mathrm{PV} \times \mathrm{pcb} \times 0.35 \\
& -\mathrm{NM} \times \mathrm{cm}-\mathrm{NP} \times \mathrm{cp}
\end{aligned}
$$

Fórmula 2. Ecuación de ganancias escenario económico 1.

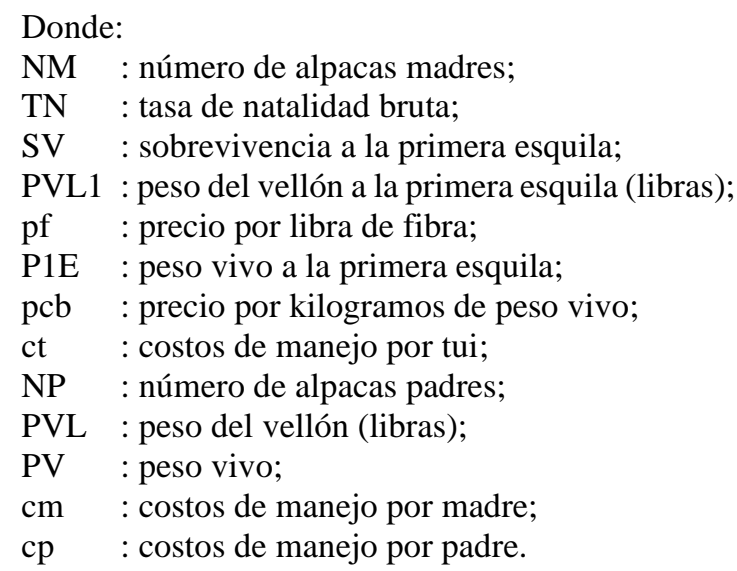

La Fórmula 2 expresa los ingresos y costos en función de los caracteres biológicos para un rebaño estabilizado de alpacas en el escenario 1. Los ingresos fueron definidos por categoría de alpaca, por la venta de fibra blanca al barrer y alpacas en pie con destino de carne:

Tui: NM $\times$ TN $\times$ SV $($ PVL1 $\times p f+P 1 E \times p c b \times 0.50)$

Madre: NM (PVL $\times$ pf + PV $\times p c b \times 0.25)$

Padre: NP $(\mathrm{PVL} \times \mathrm{pf}+\mathrm{PV} \times \mathrm{pcb} \times 0.35)$

Asimismo, los costos fueron definidos por categoría de alpaca y actividades de manejo:

Destete, esquila: Tui: $\mathrm{NM} \times \mathrm{TN} \times \mathrm{SV} \times \mathrm{ct}$

Alimentación, empadre y parición, esquila:

Madre: $\mathrm{NM} \times \mathrm{cm}$

Esquila y selección de reemplazos: Padre: NP× cp $\underline{\text { La ecuación de ganancias del escenario económico } 2 \text { es }}$ definida según la Fórmula 3.

Los caracteres biológicos relacionados a los tuis fueron SV, PVL1, P1E y el diámetro de fibra a la primera esquila (DF1E), los caracteres relacionados a los padres y madres fueron PVL, PV y el diámetro de fibra en alpacas adultas (DF).

$$
\begin{aligned}
\mathrm{EC} 2=\mathrm{NM} \times \mathrm{TN} & \times \mathrm{SV}[\mathrm{PVL} 1 \times \mathrm{pf}+(\mathrm{VU}-\mathrm{DF} 1 \mathrm{E}) \mathrm{cc} \\
& +\mathrm{P} 1 \mathrm{E} \times \mathrm{pcb} \times 0.5-\mathrm{ct}] \\
& +(\mathrm{NM}+\mathrm{NP})[\mathrm{PVL} \times \mathrm{pf} \\
& +(\mathrm{VU}-\mathrm{DF}) \mathrm{cc}] \\
& +\mathrm{NM} \times \mathrm{PV} \times \mathrm{pcb} \times 0.25 \\
& +\mathrm{NP} \times \mathrm{PV} \times \mathrm{pcb} \times 0.35 \\
& -\mathrm{NM} \times \mathrm{cm}-\mathrm{NP} \times \mathrm{cp}
\end{aligned}
$$

Fórmula 3. Ecuación de ganancias escenario económico 2.
Donde:

NM : número de alpacas madres;

TN : tasa de natalidad bruta;

SV : sobrevivencia a la primera esquila;

PVL1: peso del vellón a la primera esquila (libras);

pf : precio por libra de fibra;

VU : valor umbral por categoría;

DF1E: diámetro de fibra a la primera esquila;

DF : diámetro de fibra;

cc : compensación por categoría;

P1E : peso vivo a la primera esquila;

pcb : precio por kilogramos de peso vivo;

ct : costos de manejo por tui;

NP : número de alpacas padres;

PVL : peso del vellón (libras);

PV : peso vivo;

$\mathrm{cm}$ : costos de manejo por madre;

cp : costos de manejo por padre.

La Fórmula 3 expresa los ingresos y costos en función de los caracteres biológicos para un rebaño estabilizado de alpacas en el escenario 2. A diferencia del escenario 1, los ingresos fueron definidos por categoría de alpaca, por la venta de fibra blanca categorizada y alpacas en pie con destino de carne:

Tui: $\mathrm{NM} \times \mathrm{TN} \times \mathrm{SV}(\mathrm{PVL} 1 \times \mathrm{pf}+(\mathrm{VU}-\mathrm{DF} 1 \mathrm{E}) \mathrm{cc}$ $+\mathrm{P} 1 \mathrm{E} \times \mathrm{pcb} \times 0.50)$

Madre: NM $(\mathrm{PVL} \times \mathrm{pf}+(\mathrm{VU}-\mathrm{DF}) \mathrm{cc}+\mathrm{PV} \times \mathrm{pcb}$ $\times 0.25)$

Padre: NP $(\mathrm{PVL} \times \mathrm{pf}+(\mathrm{VU}-\mathrm{DF}) \mathrm{cc}+\mathrm{PV} \times \mathrm{pcb} \times$ $0.35)$

Asimismo, los costos fueron definidos por categoría de alpaca y actividades de manejo:

Destete, esquila:Tui: $\mathrm{NM} \times \mathrm{TN} \times \mathrm{SV} \times \mathrm{ct}$

Alimentación, empadre y parición, esquila:Madre: $\mathrm{NM} \times \mathrm{cm}$

Esquila y selección de reemplazos:Padre: $\mathrm{NP} \times \mathrm{cp}$

d. Valores relativos económicos.

El valor relativo económico (VRE) de cada carácter biológico fue estimado mediante las derivadas parciales de las ecuaciones de ganancias, utilizando la hoja de cálculo de Microsoft Excel. En los cálculos se consideraron los mismos parámetros productivos, reproductivos, ingresos y costos utilizados en las ecuaciones de ganancias. El VRE expresó el beneficio obtenido por el incremento del valor genético de un carácter en una unidad, cuando los demás caracteres permanecen en magnitud constante (Bohan et al., 2019). Asimismo, para determinar la influencia de la forma de pago de la fibra sobre el VRE, se realizó la comparación entre los escenarios económicos 1 y 2.

e. Definición del objetivo de selección.

El objetivo de selección fue definido según el análisis de la heredabilidad, el VRE y la facilidad de medición del carácter biológico. Esta evaluación se realizó de manera individual considerando un carácter a la vez, analizando en primer lugar la heredabilidad, dado que es un factor importante para la repuesta a la selección; en segundo lugar, el VRE; y, en tercer lugar, 
la facilidad de medición del carácter. Así, los caracteres seleccionados en el objetivo de selección tendrían una heredabilidad media a alta, un VRE favorable a los productores y serían fáciles de registrar o medir en campo. El objetivo de selección para los escenarios económicos 1 y 2 se expresó bajo la forma de genotipo agregado (Hazel, 1943), en función de los VRE y valores genéticos aditivos de los caracteres biológicos.

\section{Resultados y discusión}

\section{Crianza de alpacas en la CCSPR}

La crianza de alpacas en la CCSPR es extensiva, es decir, con una alimentación a base de pastizales tipo pajonal. Las tierras de pastoreo tienen una extensión de 4275.8 ha, de las cuales 4189.4 ha corresponden a áreas en condición buena y 86.4 ha en condición regular para alpacas, con una tendencia positiva y donde destaca la presencia de gramíneas altas perennes, tales como Festuca dolichophylla, Festuca orthophylla, Stipa ichu, Calamagrostis antoniana y Calamagrostis rigida. Los pastizales son pastoreados con una capacidad de carga de 1.5 unidades alpacas.ha ${ }^{1}$, utilizando distintos sistemas de pastoreo rotativos, y para ello los animales son agrupados en lotes y/o puntas de animales (parición: madres y crías, empadre: padres y madres, tuis: tuis menores y mayores) para facilitar el manejo, la vigilancia y programar los días de pastoreo a fin de cubrir las necesidades de alimentación diferenciadas.

El CPA de la CCSPR fue 2933.40 alpacas para el periodo $2007-2010$ y 2565.33 alpacas para el periodo 2014-2016, mostrando una reducción de 368.07 alpacas para el CPA entre los años 2007 y 2016. La NB fue de $65.51 \%$ y $56.77 \%$ para los periodos de evaluación 2007-2010 y 2014-2016, respectivamente. La mortalidad promedio fue de $4.69 \%$ y $7.78 \%$ para los periodos 2007-2010 y 2014-2016, respectivamente, donde las crías fueron la clase con mayor mortalidad (73 crías muertas por año). La saca más alta fue de $45.02 \%$ para el año 2016, debido a la tendencia de estabilizar el tamaño del rebaño produciendo saca forzada, principalmente de tuis machos menores de 1 año de edad. La CCSPR no registró compra de animales, dado que fueron generando sus propios reemplazos, por tanto, el incremento bruto y real fue similar. La EG del manejo de alpacas promedio para el periodo $2007-2010$ fue $25.21 \%$ y para el periodo 2014 2016 fue 25.4, siendo similar entre los años 2007 al 2016 (Tabla 1).

Los índices técnicos evidencian un buen nivel tecnológico y se corresponden con los estándares reportados por Gutiérrez et al. (1993) y Ruiz et al. (2015). Estos resultados demostraron que la población se encuentra estabilizada y que el manejo técnico fue estable debido a que no se reportaron variaciones significativas en la eficiencia ganadera. Por lo tanto, sería factible la implementación de registros de producción y genealogía para el desarrollo de un programa de mejora genética de alpacas en la CCSPR.

Los costos considerados fueron los provenientes de las actividades de administración y manejo ganadero. Los costos fijos fueron los costos de administración y fueron expresados en base a una unidad alpaca (UAL) por año. El costo fijo más bajo fue $\mathrm{S} / 84.62$ para el año 2008 y el más alto fue S/425.44 para el año 2016; siendo en promedio $\mathrm{S} / 271.79 \cdot \mathrm{UAL}^{-1} \cdot \mathrm{año}^{-1}$ para el periodo de evaluación 2006-2016.

Los costos variables fueron calculados por las actividades de manejo. El costo de alimentación fue de $\mathrm{S} / 54.64 \cdot \mathrm{UAL}^{-1} \cdot \mathrm{anno}^{-1}$, incluyendo costos por uso de pastizales y pastores con un sueldo mínimo de S/930.00. El costo de empadre y parición fue de $\mathrm{S} / 9.77 \cdot \mathrm{UAL}^{-1} \cdot \mathrm{año}^{-1}$, incluyendo los costos de insumos veterinarios y mano de obra temporal por tres meses con un sueldo mínimo de $\mathrm{S} / 930.00$. El costo de destete por tui fue $\mathrm{S} / 7.74 \cdot \mathrm{anno}^{-1}$ considerando los insumos veterinarios y mano de obra temporal por un mes con sueldo mínimo de S/930.00 para el tratamiento sanitario y apoyo en el manejo de los tuis. El costo de esquila fue $\mathrm{S} / 5.92 \cdot \mathrm{UAL}^{-1} \cdot \mathrm{anno}^{-1}$ considerando el pago por jornal diario de $\mathrm{S} / 50.00$ a los ayudantes, envellonadores y el pago al destajo de $\mathrm{S} / 3.50$ a los esquiladores por alpaca. El costo de selección de reemplazos fue $\mathrm{S} / 15.50 \cdot \mathrm{UAL}^{-1} \cdot \mathrm{año}^{-1}$ incluyendo los análisis de finura de la fibra y el honorario de S/1 500.00 para el personal técnico que realice la evaluación y clasificación del rebaño. El costo variable total fue $\mathrm{S} / 93.57 \cdot \mathrm{UAL}^{-1} \cdot \mathrm{año}^{-1}$ considerando todas las actividades de manejo.

Los costos de producción por UAL reportados en los balances contables de la CCSPR durante el periodo 2006-2016 son presentados en la Tabla 2. Al realizar la comparación entre los costos variables estimados y lo reportado en los balances contables de la cooperativa comunal en el año 2016, se encontró una diferencia de S/6.70, lo cual validó los costos variables estimados para el cálculo de los VRE.

Los ingresos fueron generados por la venta de fibra y alpacas en pie. Durante el periodo 2006-2016, la proporción de los ingresos fue de $45.09 \%$ por venta de fibra, $53.92 \%$ por venta de alpacas en pie, $0.5 \%$ por venta de carne de alpaca y $0.49 \%$ por venta de pieles de alpaca. Asimismo, al analizar el periodo 2010-2016, los ingresos por alpacas en pie fueron mayores, representando más del $60 \%$ de los ingresos totales en los años 2011 y 2016 (Tabla 3), lo cual demuestra que la venta de fibra no siempre fue el principal ingreso de la CCSPR y que el productor se vio involucrado en diferentes escenarios económicos durante el periodo 2006-2016 debido a los precios y forma de pago de la fibra de alpaca.

Los precios promedios de la fibra y alpacas en pie registrados en el año 2016 por la CCSPR fueron los siguientes: $\mathrm{S} / 10.00 \cdot \mathrm{lb}^{-1}$ de fibra blanca, $\mathrm{S} / 200.00$ por alpaca tui en pie con destino a carne y $\mathrm{S} / 250.00$ por 
alpaca padre o madre en pie con destino a carne. Durante el periodo 2006-2016 se presentaron disminuciones temporales en el precio de fibra tanto a nivel nacional como internacional, en este contexto la venta de alpacas en pie fue un rubro importante para mantener la rentabilidad de la crianza de alpacas de la CCSPR cuando los precios de la fibra fueron bajos. Esto demostró la importancia del manejo de los pastizales y de los animales durante el empadre, parición y destete, siendo actividades claves en la producción de alpacas para la venta en pie.

Valores Relativos Económicos (VRE)

En el escenario económico 1, los VRE fueron 75.62, 10.60, 5.85, 1.67, 1.55 soles para la SV, PVL, PVL1, P1E y PV, respectivamente. La SV fue el carácter de mayor valor económico por unidad del carácter en este escenario. El P1E y PV tuvieron similares valores económicos, siendo mayor el P1E en solo $\mathrm{S} / 0.12$, lo cual evidenció que la evaluación del peso vivo en edad adulta no sería prioritaria. El PV fue superior al PVL1 en S/4.75; por tanto, sería importante mantener o priorizar la evaluación del PV en edad adulta (Tabla 4).

En el escenario económico 2, los caracteres de SV, DF1E y DF, estuvieron directamente relacionados al pago por fibra categorizada, por tanto, los VRE de estos caracteres fueron expresados en las categorías extrafina, fina y semifina. La SV tuvo mayor valor económico por unidad del carácter con S/71.35 y $\mathrm{S} / 69.75$ para las categorías extrafina y fina en vellones de tuis. El DF tuvo valores de $-2.12,-1.06$ y $-\mathrm{S} / 0.53$ soles para las categorías extrafina, fina y semifina en vellones de alpacas adultas. El DF1E tuvo valores respectivos de $-\mathrm{S} / 1.17$ y $-\mathrm{S} / 0.59$ para las categorías extrafina y fina en vellones de tuis. El DF tuvo mayores valores para las categorías extrafinas y fina en comparación con el DF1E; lo cual indicaría la importancia de mantener las evaluaciones de la finura hasta la edad adulta de las alpacas. Los VRE del DF y DF1E tuvieron magnitud negativa, lo cual sería favorable para el afinamiento de la fibra (Tabla 5).

Los VRE del PV, PVL1, P1E y PV fueron 10.60, $5.85,1.67$ y 1.55 soles, respectivamente. El P1E tuvo un valor similar al PV, siendo la diferencia entre ambos de S/0.12. Por tanto, se debe priorizar la evaluación del $\mathrm{P} 1 \mathrm{E}$, considerando que la venta de alpacas en pie corresponde en gran parte a la saca de tuis y fue una importante fuente de ingreso de la CCSPR.

Los caracteres de producción expresados en edad adulta (DF y PVL) tuvieron mayores valores económicos que los expresados en tuis a la primera esquila (DF1E y PVL1). Al disminuir el mérito genético del DF en 1 micra se incrementaría el beneficio económico en $\mathrm{S} / 2.12$ para la categoría extrafina y al incrementar el mérito genético del PV en 1 libra se incrementaría el beneficio económico en S/10.6.
Comparación de los VRE entre los escenarios económicos 1 y 2

La SV en los escenarios 1 y 2 muestran valores similares diferenciándose solo en S/4.27. Respecto a los caracteres PVL1, PVL, P1E y PV los valores económicos fueron iguales en ambos escenarios económicos, lo cual muestra que el pago por fibra categorizada no afectó al valor económico de estos caracteres y tampoco influyó de manera significativa en la SV (Tabla 6). Al comparar los escenarios 1 y 2, encontramos que el escenario 2 es el que más beneficio ofrece al productor, al recibir un pago por fibra categorizada sin disminuir o afectar los ingresos por los caracteres de PV, PVL1, PVL, P1E y PV. El total del beneficio por la venta de fibra categorizada fue de $\mathrm{S} / 5.46$ al considerar los valores de DF1E y DF para las categorías extra fina, fina y semifina.

El PV obtuvo mayor valor económico en ambos escenarios, siendo superior en $\mathrm{S} / 2.22$ respecto a lo reportado por Alfonso et al. (2011) para alpacas en Huancavelica. Respecto al DF el valor fue menor a los -S/7.05 reportado por Alfonso et al. (2011). Nuestros resultados fueron similares a lo encontrado por McGregor (2006), donde el VRE total fue directamente proporcional al incremento del PVL y disminuyó al incrementar el DF por encima de las 23 micras para alpacas en Australia. Asimismo, indicar que Gutiérrez et al. (2014) reportaron valores económicos similares para alpacas, pero con una metodología diferente; por tanto, no fue posible realizar la comparación.

No existe suficiente información en la literatura referida al VRE para alpacas para una adecuada discusión, pero cabe mencionar que la metodología empleada en este estudio ha sido aplicada en otras especies como vacunos (Fuerst et al., 2016), ovinos (Bohan et al., 2019) y peces (Besson et al., 2020); asimismo, dado que la información requerida para la formulación de ecuaciones de ganancias y derivación de VRE es más factible de obtener en el campo, las ecuaciones de ganancias y los VRE serían herramientas importantes para la toma de decisiones del productor alpaquero, tal como lo destaca Alfonso (2016).

Existen otras metodologías para la estimación de los VRE como los modelos bioeconómicos, que se aplican por ejemplo en peces (Janssen et al., 2017) y vacunos (Schmidtmann, 2021); sin embargo, su aplicación requiere de una evaluación y actualización constante de registros de producción, comercialización y genealogía; lo cual hace complicado su aplicación en la crianza de alpacas de la CCSPR.

Objetivo de selección

La SV fue evaluada considerando las referencias de heredabilidad de la sobrevivencia al destete, con un valor de $0.10 \pm 0.17$ (Bustinza et al., 1988) y la sobrevivencia a los 15 días con un valor de 0.02 (Cruz et al., 2015); según esto, la SV tendría baja respuesta a la selección en un programa de mejora genética. 
Además, la SV fue considerada como un carácter de difícil medición en el campo, dado que se necesitaría la implementación de registros de producción y personal calificado para el procesamiento y análisis de los datos. A pesar de que la SV obtuvo el mayor VRE, por su baja heredabilidad y poca facilidad de medición se descartó su inclusión en el objetivo de selección para los escenarios económicos 1 y 2.

El objetivo de selección para el escenario económico 1, fue definido por el PVL, PVL1 y P1E. El PV presentó reportes de heredabilidad variables, desde valores bajos de $0.098 \pm 0.016$ (Gutiérrez et al., 2008) a valores altos de $0.63 \pm 0.22$ (Wuliji et al., 2000); lo cual indicó la variación genética del carácter y una respuesta favorable a la selección. Este carácter fue considerado de fácil medición, debido a que puede ser medido en el campo utilizando de balanzas y su medición no requiere capacitación especializada.

El PVL1, presentó reportes de heredabilidad variables desde $0.38 \pm 0.34$ (Mamani, 1991) hasta 0.83 \pm 0.35 (Ponzoni et al., 1999); dada esta variabilidad genética se obtendría una respuesta favorable a la selección. Además, considerando su facilidad de medición y su asociación con la forma de pago de la fibra, el PVL1 fue incluido en el objetivo de selección. Asimismo, el P1E presentó reportes heredabilidad de $0.32 \pm 0.23$ (Ruiz de Castilla et al., 1992) y $0.41 \pm 0.47$ (Wuliji et al., 2000); por tanto, tendría una respuesta favorable a la selección. El VRE del P1E fue mayor al VRE del PV y dada su fácil medición fue incluido en el objetivo de selección (Tabla 7).

El objetivo de selección para el escenario económico 1, expresado en términos de genotipo agregado según Hazel (1943), fue determinado según la Fórmula 4, cuyo resultado se interpretó como el incremento del beneficio económico de la crianza de Alpacas en la CCSPR a través de la mejora genética del peso de vellón, peso de vellón a la primera esquila y peso vivo a la primera esquila.

$\mathrm{H} 1=10.6 \times \mathrm{G}_{\mathrm{PVL}}+5.85 \times \mathrm{G}_{\mathrm{PVL} 1}+1.67 \times \mathrm{G}_{\mathrm{P} 1 \mathrm{E}}$

Fórmula 4. Objetivo de selección para el escenario económico 1.

Donde:

H1 : valor del genotipo agregado para el escenario económico 1 ;

$\mathrm{G}_{\mathrm{PVL}}$ : valor genético aditivo del carácter peso del vellón;

GPVL1: valor genético aditivo del peso del vellón a la primera esquila;

$\mathrm{G}_{\mathrm{P} 1 \mathrm{E}}$ : valor genético aditivo del peso vivo a la primera esquila.

El objetivo de selección para el escenario económico 2, fue definido por el PVL, PVL1 y el DF. El PVL fue analizado según los reportes de heredabilidad de Gutiérrez et al. (2008) con un valor de
$0.098 \pm 0.016$ y Wuliji et al. (2000) con un valor de $0.63 \pm 0.22$, lo cual evidenció una respuesta favorable a la selección. El PVL y PVL1 fueron incluidos en el objetivo de selección por su facilidad de medición y mayor VRE en el escenario 2. Respecto al DF se reportaron valores de heredabilidad de $0.412 \pm 0.015$ (Gutiérrez et al., 2008) y $0.369 \pm 0.012$ (Cervantes et al., 2010), lo cual demostró una respuesta favorable a la selección. Asimismo, el DF1E fue desestimado del objetivo de selección debido a su menor VRE respecto al DF (Tabla 8).

El objetivo de selección para el escenario económico 2, expresado en términos de genotipo agregado según Hazel (1943), fue determinado según la Fórmula 5, cuyo resultado se interpretó como el incremento del beneficio económico de la crianza de Alpacas en la CCSPR a través de la mejora genética del peso de vellón, peso de vellón a la primera esquila y diámetro de fibra.

$\mathrm{H} 2=10.6 \times \mathrm{G}_{\mathrm{PVL}}+5.85 \times \mathrm{G}_{\mathrm{PVL} 1}-2.12 \times \mathrm{G}_{\mathrm{DF}}$

Fórmula 5. Objetivo de selección para el escenario económico 2.

Donde:

H2 : valor del genotipo agregado para el escenario económico 2;

GPVL : valor genético aditivo del carácter peso del vellón;

GPVL1: valor genético aditivo del peso del vellón a la primera esquila;

$\mathrm{G}_{\mathrm{DF}}$ : valor genético aditivo del diámetro de fibra.

No se ha encontrado en la literatura referencias de objetivos de selección para alpacas que incluyan los escenarios económicos en los que se vean involucrados los productores alpaqueros; sin embargo, este enfoque ya ha sido empleado en vacunos (Fuerst et al., 2016) donde también se consideraron los escenarios económicos en la definición de objetivos de selección para ganado Fleckvieh y Brown Swiss en Austria. Por lo tanto, si consideramos los diferentes escenarios económicos en la definición de los objetivos de selección en un rebaño de alpacas, se podría contar con una herramienta acorde con las circunstancias de producción y comercialización de la fibra.

\section{Conclusiones}

La crianza de alpacas de la Cooperativa Comunal San Pedro de Racco (CCSPR) evidenció un buen manejo técnico y una población estabilizada de alpacas, según la eficiencia ganadera y capital promedio anual. El pago por fibra categorizada favoreció la magnitud del valor relativo económico del diámetro de fibra y diámetro de fibra a la primera esquila, contribuyendo al afinamiento de los vellones. El peso de vellón fue el carácter de mayor valor relativo económico en los objetivos de selección para los escenarios económicos 
1 y 2. El escenario económico 2 fue el más beneficioso para el productor alpaquero debido a que el pago por fibra categorizada no afectó el valor relativo económico del peso de vellón, peso de vellón a la primera esquila y peso vivo.

\section{Literatura citada}

Alfonso L., Buritica J., Quispe R. \& Quicaño I. 2011. Derivation of economic values for fibre diameter fleece weight in alpacas. In: Pérez-Cabal M.Á., Gutiérrez J.P., Cervantes I. \& Alcalde M.J. (eds). Fibre production in South American camelids and other fibre animals. 145150. Wageningen Academic Publishers, Wageningen. DOI: https://doi.org/10.3920/978-90-8686-727-1_19.

Alfonso L. 2016. Technical note: An approach to derive breeding goals from the preferences of decision makers. Journal of Animal Science, 94(11): 4498-4506. DOI: https://doi.org/10.2527/jas.2016-0685.

Besson M., Komen H., Rose G. \& Vandeputte M. 2020. The genetic correlation between feed conversion ratio and growth rate affects the design of a breeding program for more sustainable fish production. Genetics Selection Evolution, 52: art. 5. DOI: https://doi.org/10.1186/s12711-020-0524-0.

Bohan A., Shalloo L., Creighton P., Berry D.P., Boland T.M., O'Brien A.C., Pabiou T., Wall E., McDermott K. \& McHugh N. 2019. Deriving economic values for national sheep breeding objectives using a bio-economic model. Livestock $\quad$ Science, 227 : 44-54 DOI: https://doi.org/10.1016/j.livsci.2019.05.018.

Bustinza A.V., Burfening P.J. \& Blacwell R.L. 1988. Factors Affecting Survival in Young Alpacas (Lama pacos). Journal of Animal Science, 66(5): 1139-1143. DOI: https://doi.org/10.2527/jas1988.6651139x.

Cervantes I., Perez-Cabal M.A., Morante R., Burgos A., Salgado C., Nieto B., Goyache F. \& Gutiérrez J.P. 2010. Genetic parameters and relationship between fibre and type traits in two breeds of Peruvian alpacas. Small Ruminant Research, 88(1): 6-11. DOI: https://doi.org/10.1016/j.smallrumres.2009.10.016.

Cruz A., Burgos A., Morante R., Cervantes I. \& Gutiérrez J. 2015. Importancia de la estima de parámetros genéticos de caracteres productivos y funcionales en alpacas. VII Congreso Mundial de Camélidos Sudamericanos. PunoPerú.

Fuerst-Waltl B., Fuerst C., Obritzhauser W. \& Egger-Danner C. 2016. Sustainable breeding objectives and possible selection response: Finding the balance between economics and breeders' preferences. Journal of Dairy Science, $\quad$ 99(12): 9796-9809. DOI: https://doi.org/10.3168/jds.2016-11095.

Germaná C., Chaquilla O., Santos G., Ferrari M., Krusich C. \& Kindgard F. 2016. Estudio socioeconómico de los pastores andinos de Perú, Ecuador, Bolivia y Argentina. Grupo Voluntario Civil Arequipa, Perú. Primera edición. Editorial El Alva.

Gutiérrez G. 2011. Valores Estimados de los Parámetros Genéticos en Poblaciones de Alpacas. En: Producción y Tecnología en Camélidos Sudamericanos. 241-249. Universidad Nacional de Huancavelica.

Gutiérrez G.A., Flores E.R., Trejo W.E., Tellez J. \& Zarate A. 1993. Manual: producción de alpacas y tecnología de sus productos. Universidad Nacional Agraria La Molina / Proyecto TTA. Lima, Perú.
Gutiérrez J.P., Cervantes I., Pérez-Cabal M.A., Burgos A. \& Morante R. 2014. Weighting Fibre and Morphological Traits in a Genetic Index for an Alpaca Breeding Programme. Animal, 8(3): 360-369. DOI: $10.1017 / \mathrm{S} 1751731113002358$.

Gutiérrez J.P., Goyache F., Burgos A. \& Cervantes I. 2008. Genetic analysis of six production traits in Peruvian alpacas. Livestock Science, 123(2-3): 193-197. DOI: 10.1016/j.livsci.2008.11.006.

Gutiérrez G.A., Gutiérrez J.P., Huanca T. \& Wurzinger M. 2019. Challenges and opportunities of genetic improvement in alpacas and llamas in Peru. Conference: 11th World Congress on Genetics Applied to Livestock Production, February 12-16, 2018. In: Proceedings of the World Congress on Genetics Applied to Livestock Production, 11.762. Auckland, Nueva Zelanda. http://www.wcgalp.org/proceedings/2018/challengesand-opportunities-genetic-improvement-alpacas-andllamas-peru.

Hazel L.N. 1943. The genetic basis for constructing selection indexes. Genetics, 28(6): 476-490. https://www.genetics.org/content/28/6/476.

INEI. 2012. IV Censo Nacional Agropecuario. INEI (Instituto Nacional de Estadística e informática). Perú. http://censos.inei.gob.pe/cenagro/tabulados/.

Janssen K., Berentsen P., Besson M. \& Komen H. 2017. Derivation of economic values for production traits in aquaculture species. Genetics Selection Evolution, 49: art. 5. DOI: https://doi.org/10.1186/s12711-016-0278-x.

Mamani G. 1991. Parámetros genéticos del peso vivo y vellón en alpacas Huacaya de La Raya, Puno. En: VII Conv. Int. Especialistas en Cam. Sud., Jujuy, Argentina.

Mamani G. 2013. Estructura genética poblacional y tendencia genética de peso vivo al nacimiento en alpacas del banco de germoplasma de Quimsachata del INIA en Puno. Tesis para optar el grado de magister scientiae en producción animal. Universidad Nacional Agraria La Molina. http://repositorio.lamolina.edu.pe/handle/UNALM/2237.

McGregor B.A. 2006. Production, attributes and relative value of alpaca fleeces in southern Australia and implications for industry development. Small Ruminant Research, 61(2-3), 93-111. DOI: https://doi.org/10.1016/j.smallrumres.2005.07.001.

MINAGRI. 2015. Dinámica agropecuaria 2004-2013. Compendio elaborado por la Dirección General de Seguimiento y Evaluación de Políticas. MINAGRI (Ministerio de Agricultura y Riego). Perú. https://sinia.minam.gob.pe/documentos/dinamicaagropecuaria-2004-2013.

Nielsen H.M., Amer P.R. \& Byrne T.J. 2014. Approaches to formulating practical breeding objectives for animal production systems. Acta Agriculturae Scandinavica, Section A - Animal Science, 64(1: A special issue in honor of Professor Odd Vangen): 2-12. DOI: https://doi.org/10.1080/09064702.2013.827237.

Ponzoni R.W. 1986. A profit equation for the definition of the breeding objective of Australian merino sheep. Journal of Animal Breeding and Genetics, 103(1-5): 342-357. DOI: $10.1111 /$ j.1439-0388.1986.tb00096.x. 
Ponzoni R.W., Grimson R.J., Hill J.A., Hubbard D.J., McGregor B.A., Howse A., Carmichael I. \& Judson G.J. 1999. The inheritance of and association among some production traits in young Australian alpacas. Proc. Assoc. Advmt. Anim. Breed. Genet., 13: 468-471. http://www.aaabg.org/livestocklibrary/1999/AB99111.p df.

Roque J.R. 2019. Estimación de heredabilidad de peso vivo y longitud de mecha en alpacas Huacaya. Aporte Santiaguino, $\quad 12(2)$ : 161-173. DOI: https://doi.org/10.32911/as.2019.v12.n2.639.

Ruiz De Castilla M., Alagon G. \& Quirita C.R. 1992. Estudio de parámetros genéticos en Alpacas Huacaya (Genetic parameter study in Alpacas Huacaya). En: Informe de trabajos de investigación en Alpacas y Llamas de color. Vol. II (genética). 1-28.

Ruiz J.A., Gutiérrez G.A. \& Flores E.R. 2015. Índices pecuarios de tres unidades de producción de alpacas en Pasco. VII Congreso Mundial de Camélidos Sudamericanos. Puno-Perú.

Salvá B.K., Zumalacárregui J.M., Figueira A.C., Osorio M.T \& Mateo J. 2009. Nutrient composition and technological quality of meat from alpacas reared in Peru. Meat Science 82(4): 450-455. DOI: 10.1016/j.meatsci.2009.02.015.
Schmidtmann C., Thaller G., Kargo M., Hinrichs D. \& Ettema J. 2021. Derivation of economic values for German dairy breeds by means of a bio-economic model - with special emphasis on functional traits. Journal of Dairy Science, 104(3): 3144-3157. DOI: https://doi.org/10.3168/jds.2019-17635.

SENAMHI. 2015. Precipitación total anual, según departamento, 2000-2015. SENAMHI (Servicio Nacional de Meteorología e Hidrología). Perú. Fecha de consulta: $10 \quad$ de Junio 2015. https://www.senamhi.gob.pe/?p=descarga-datoshidrometeorológicos.

Workman J.P. 1981. Analyzing ranch income statements - a modified approach. Rangelands, 3(4): 146-148. DOI: http://hdl.handle.net/10150/638292.

Wuliji T., Davis G.H., Dodds K.G., Turner P.R., Andrews R.N. \& Bruce G.D. 2000. Production performance, repeatability and heritability estimates for live weight, fleece weight and fiber characteristics of alpacas in New Zealand. Small Ruminant Research 37(3), 189-201. DOI: $10.1016 / \mathrm{s} 0921-4488(00) 00127-9$.

Tabla 1. Índices técnicos de la CCSPR para los periodos 2007-2010 y 2014-2016.

\begin{tabular}{cccccccc}
\hline $\begin{array}{c}\text { Índices } \\
\text { técnicos }\end{array}$ & $\mathbf{2 0 0 7}$ & $\mathbf{2 0 0 8}$ & $\mathbf{2 0 0 9}$ & $\mathbf{2 0 1 0}$ & $\mathbf{2 0 1 4}$ & $\mathbf{2 0 1 5}$ & $\mathbf{2 0 1 6}$ \\
\hline CPA & 2513.62 & 2748.92 & 3070.92 & 3400.15 & 2440.00 & 2848 & 2408 \\
$\% \mathrm{NB}$ & 66.22 & 65.19 & 77.05 & 65.58 & 51.18 & 64.22 & 54.9 \\
$\% \mathrm{NR}$ & 29.32 & 30.59 & 32.69 & 28.53 & 33.77 & 33.85 & 32.35 \\
$\% \mathrm{~S}$ & 12.17 & 16.04 & 19.80 & 25.59 & 35.17 & 25.49 & 45.02 \\
$\% \mathrm{M}$ & 4.14 & 5.20 & 3.84 & 5.59 & 7.71 & 5.79 & 9.84 \\
IB & 277 & 264 & 285 & -86 & -188.00 & -45 & -486 \\
IR & 277 & 264 & 285 & -90 & -190.00 & -49 & -486 \\
\%IR & 11.02 & 9.60 & 9.28 & -2.65 & -7.79 & -1.72 & -20.18 \\
\%EG & 23.19 & 25.65 & 29.08 & 22.94 & 27.46 & 23.91 & 24.83 \\
\hline
\end{tabular}

Tabla 2. Costos por unidad alpaca (UAL), periodo 20062016.

\begin{tabular}{ccccc}
\hline Año & $\begin{array}{c}\text { Unidades } \\
\text { alpaca } \\
\text { (UAL) }\end{array}$ & $\begin{array}{c}\text { Costos de } \\
\text { producción } \\
\text { de fibra } \\
\text { por UAL }\end{array}$ & $\begin{array}{c}\text { Costo de } \\
\text { Producción } \\
\text { por UAL }\end{array}$ & $\begin{array}{c}\text { Costo de } \\
\text { Ventas } \\
\text { por UAL }\end{array}$ \\
\hline 2006 & 1409 & - & 22.95 & - \\
2007 & 1464 & - & 8.80 & - \\
2008 & 1668 & - & 9.28 & - \\
2009 & 1763 & - & 47.85 & - \\
2010 & 1649 & - & 57.12 & - \\
2011 & 1688 & - & 43.70 & - \\
2012 & 1795 & - & 77.19 & - \\
2013 & 1628 & 1.69 & 49.01 & 88.59 \\
2014 & 1577 & 5.29 & 51.64 & 68.22 \\
2015 & 1405 & 7.56 & 61.92 & 74.87 \\
2016 & 1124 & 9.89 & 76.99 & 160.11 \\
\hline
\end{tabular}




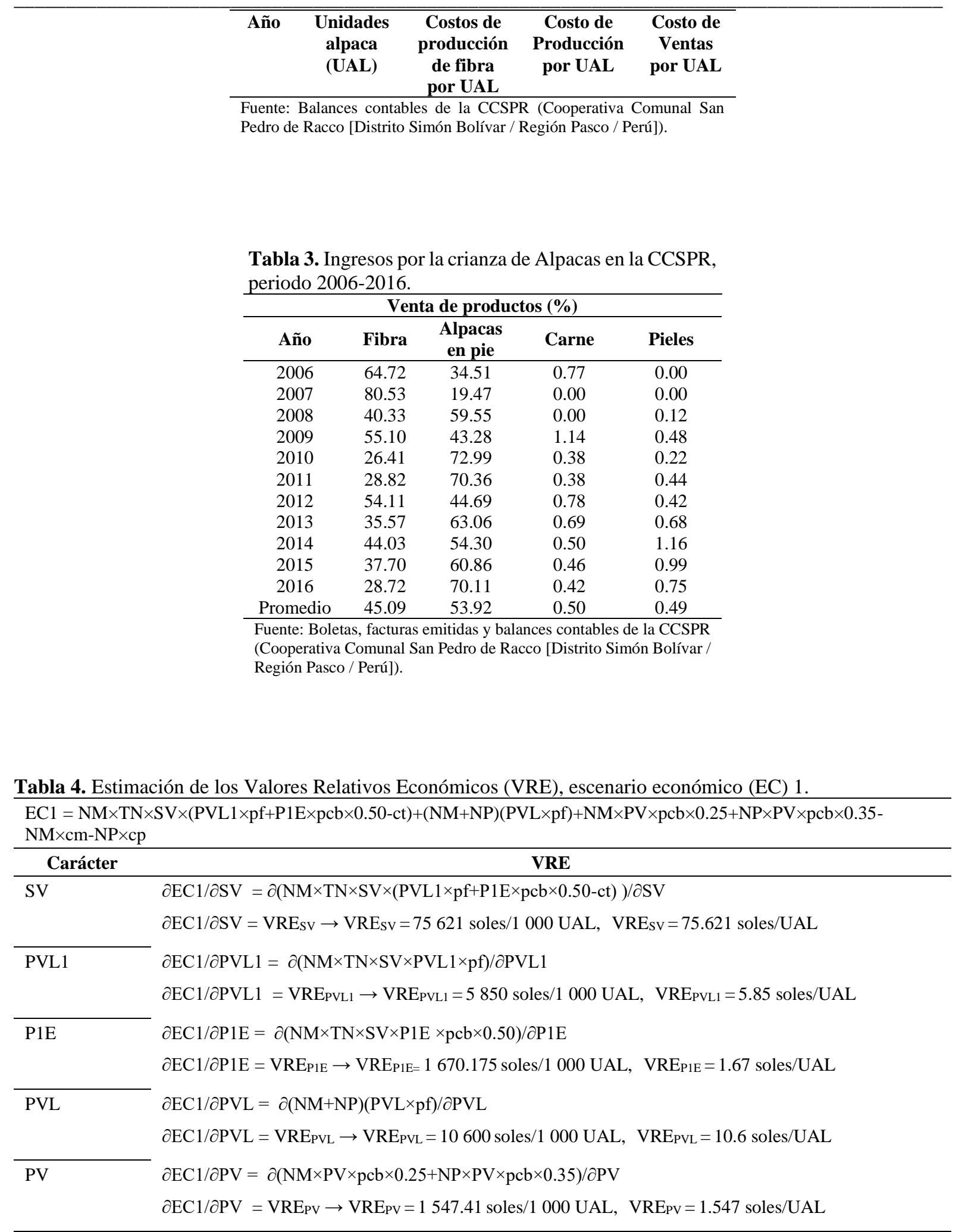

NM: número de alpacas madres; TN: tasa de natalidad bruta; SV: sobrevivencia a la primera esquila; PVL1: peso del vellón a la primera esquila (libras); pf: precio por libra de fibra; P1E: peso vivo a la primera esquila; pcb: precio por kilogramos de peso vivo; ct: costos de manejo por tui; NP: número de alpacas padres; PVL: peso del vellón (libras); PV: peso vivo; cm: costos de manejo por madre; cp: costos de manejo por padre. UAL: Unidad de alpaca. 
Tabla 5. Estimación de los Valores Relativos Económicos (VRE), escenario económico (EC) 2.

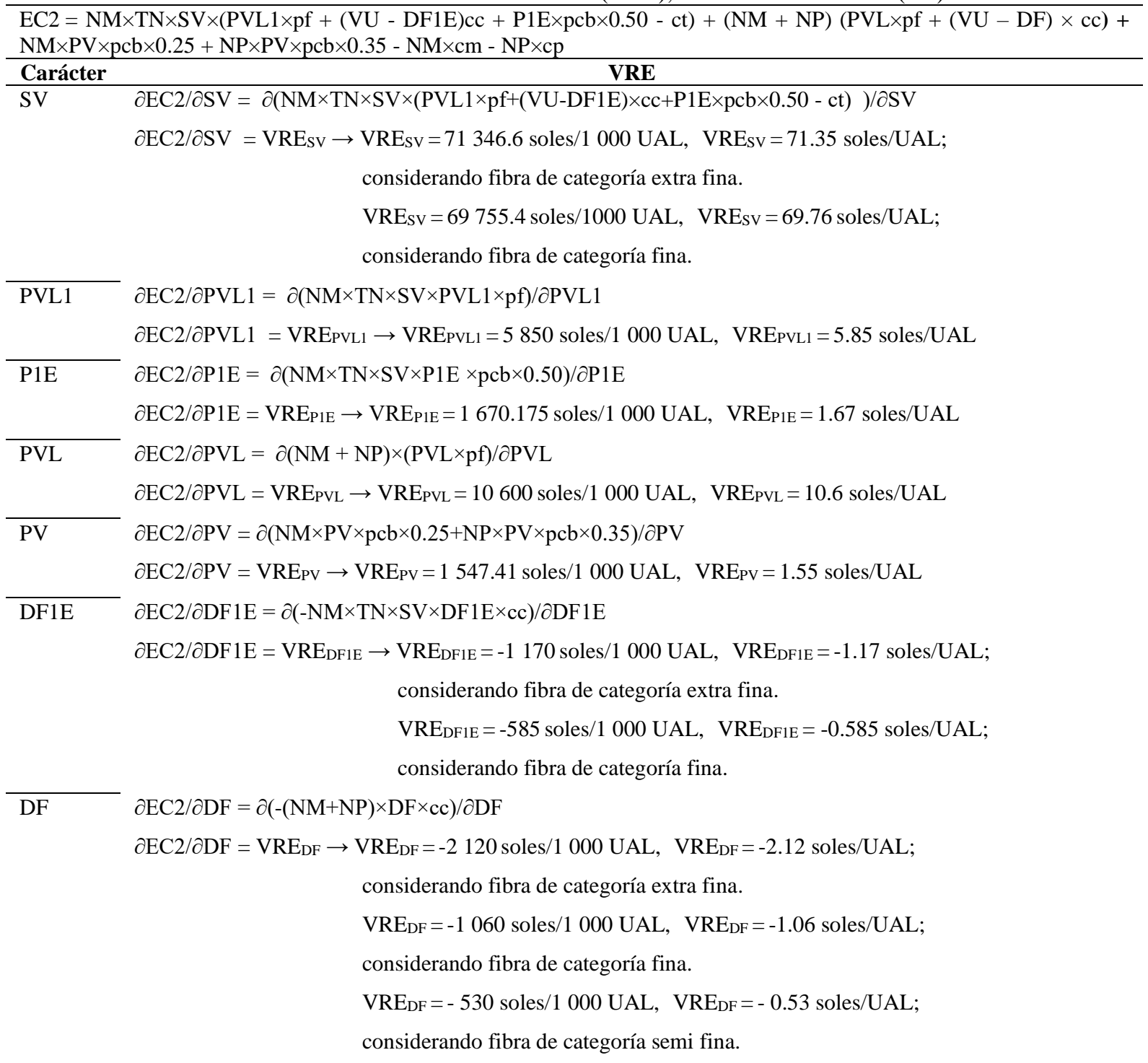

NM: número de alpacas madres; TN: tasa de natalidad bruta; SV: sobrevivencia a la primera esquila; PVL1: peso del vellón a la primera esquila (libras); pf: precio por libra de fibra; VU: valor umbral por categoría; DF1E: diámetro de fibra a la primera esquila; DF: diámetro de fibra; cc: compensación por categoría; P1E: peso vivo a la primera esquila; pcb: precio por kilogramos de peso vivo; ct: costos de manejo por tui; NP: número de alpacas padres; PVL: peso del vellón (libras); PV: peso vivo; cm: costos de manejo por madre; cp: costos de manejo por padre. UAL: Unidad de alpaca. 
Tabla 6. Comparación de los Valores Relativos Económicos (VRE) entre los escenarios económicos 1 y 2.

\begin{tabular}{clcc}
\multicolumn{2}{c}{ Caracteres biológicos } & $\begin{array}{c}\text { Escenario 1 } \\
\text { VRE } \\
\text { (soles/alpaca madre) }\end{array}$ & $\begin{array}{c}\text { Escenario 2 } \\
\text { VRE } \\
\text { (soles/alpaca madre) }\end{array}$ \\
\hline Sobrevivencia a & Extrafina & 75.62 & 71.35 \\
la $1^{\circ}$ esquila (SV) & Fina & - & 69.75 \\
Diámetro de fibra a la & Extra fina & - & -1.17 \\
$1^{\circ}$ esquila (DF1E) & Fina & - & -0.585 \\
Diámetro de fibra (DF) & Extra fina & - & -2.12 \\
& Fina & - & -1.06 \\
Peso de vellón a la $1^{\circ}$ esquila (PVL1) & Semifina & - & -0.53 \\
Peso de vellón (PVL) & & 10.85 & 5.85 \\
Peso vivo a la $1^{\circ}$ esquila (P1E) & 1.67 & 10.6 \\
Peso vivo (PV) & & 1.55 & 1.67 \\
\hline
\end{tabular}

Tabla 7. Definición del objetivo de selección del escenario económico 1.

\begin{tabular}{|c|c|c|c|}
\hline Caracteres biológicos & Heredabilidad & $\begin{array}{c}\text { Valor Relativo } \\
\text { Económico (VRE) } \\
\text { (soles/alpaca madre) }\end{array}$ & $\begin{array}{l}\text { Medición } \\
\text { del carácter }\end{array}$ \\
\hline $\begin{array}{l}\text { Sobrevivencia a la } \\
1^{\circ} \text { esquila (SV) }\end{array}$ & $\begin{array}{c}0.10 \pm 0.17 \\
\text { Bustinza et al. }(1988) \\
0.02 \\
\text { Cruz et al. }(2015)\end{array}$ & 75.62 & Poco mensurable* \\
\hline $\begin{array}{l}\text { Peso de vellón a la } \\
1^{\circ} \text { esquila (PVL1) }\end{array}$ & $\begin{array}{c}0.38 \pm(0.34) \\
\text { Mamani }(1991) \\
0.83 \pm 0.35 \\
\text { Ponzoni et al. }(1999)\end{array}$ & 5.85 & Mensurable** \\
\hline $\begin{array}{l}\text { Peso vivo a la } \\
1^{\circ} \text { esquila (P1E) }\end{array}$ & $\begin{array}{c}0.32 \pm 0.23 \\
\text { Ruiz de Castilla } \text { et al. (1992) } \\
0.41(0.47) \\
\text { Wuliji et al. }(2000)\end{array}$ & 1.67 & Mensurable \\
\hline Peso de vellón (PVL) & $\begin{array}{c}0.63(0.22) \\
\text { Wuliji et al. }(2000) \\
0.098 \pm 0.016 \\
\text { Gutiérrez et al. }(2008)\end{array}$ & 10.6 & Mensurable \\
\hline Peso vivo (PV) & $\begin{array}{c}0.27 \pm 0.08 \\
\text { Roque }(2019)\end{array}$ & 1.55 & Mensurable \\
\hline
\end{tabular}


Tabla 8. Definición del objetivo de selección del escenario económico 2.

\begin{tabular}{|c|c|c|c|}
\hline Caracteres biológicos & Heredabilidad & $\begin{array}{c}\text { Valor Relativo } \\
\text { Económico (VRE) } \\
\text { (soles/alpaca madre) }\end{array}$ & Medición del carácter \\
\hline $\begin{array}{c}\text { Sobrevivencia a la } 1^{\circ} \\
\text { esquila }(\mathrm{SV})\end{array}$ & $\begin{array}{c}0.10 \pm 0.17 \\
\text { Bustinza et al. }(1988) \\
0.02 \\
\text { Cruz et al. }(2015)\end{array}$ & 71.35 & Poco mensurable \\
\hline $\begin{array}{c}\text { Diámetro de fibra a la } 1^{\circ} \\
\text { esquila (DF1E) }\end{array}$ & -- & -1.17 & Mensurable \\
\hline Diámetro de fibra (DF) & $\begin{array}{c}0.412 \pm 0.015 \\
\text { Gutiérrez et al. }(2008) \\
0.369 \pm 0.012 \\
\text { Cervantes } \text { et al. } 2010\end{array}$ & -2.12 & Mensurable \\
\hline $\begin{array}{l}\text { Peso de vellón a la } 1^{\circ} \\
\text { esquila (PVL1) }\end{array}$ & $\begin{array}{c}0.38 \pm(0.34) \\
\text { Mamani }(1991) \\
0.83 \pm 0.35 \\
\text { Ponzoni et al. }(1999)\end{array}$ & 5.85 & Mensurable \\
\hline Peso de vellón (PVL) & $\begin{array}{c}0.63(0.22) \\
\text { Wuliji et al. }(2000) \\
0.098 \pm 0.016 \\
\text { Gutiérrez et al. }(2008)\end{array}$ & 10.6 & Mensurable \\
\hline $\begin{array}{l}\text { Peso vivo a la } 1^{\circ} \text { esquila } \\
\text { (P1E) }\end{array}$ & $\begin{array}{c}0.32 \pm 0.23 \\
\text { Ruiz de Castilla } \text { et al. }(1992) \\
0.41(0.47) \\
\text { Wuliji et al. }(2000)\end{array}$ & 1.67 & Mensurable \\
\hline Peso vivo (PV) & $\begin{array}{c}0.27 \pm 0.08 \\
\text { Roque }(2019) \\
\end{array}$ & 1.55 & Mensurable \\
\hline
\end{tabular}

\footnotetext{
${ }^{1}$ Departamento de Producción Animal / Facultad de Zootecnia / Universidad Nacional Agraria La Molina. Av. La Molina s/n La Molina, Lima, Casilla Lima 12, Perú.

2 jcandio@lamolina.edu.pe.

3 gustavogr@lamolina.edu.pe.
} 